\title{
Computation of iterative solutions along with stability analysis to a coupled system of fractional order differential equations
}

\author{
Sajjad Ali ${ }^{1}$, Thabet Abdeljawad ${ }^{2}$, Kamal Shah ${ }^{3 *}$, Fahd Jarad ${ }^{4}$ and Muhammad Arif ${ }^{1}$
}

\section{"Correspondence:}

kamalshah408@gmail.com

${ }^{3}$ Department of Mathematics,

University of Malakand, Khyber

Pakhtunkhwa, Pakistan

Full list of author information is

available at the end of the article

\section{Springer}

\begin{abstract}
In this research article, we investigate sufficient results for the existence, uniqueness and stability analysis of iterative solutions to a coupled system of the nonlinear fractional differential equations (FDEs) with highier order boundary conditions. The foundation of these sufficient techniques is a combination of the scheme of lower and upper solutions together with the method of monotone iterative technique. With the help of the proposed procedure, the convergence criteria for extremal solutions are smoothly achieved. Furthermore, a major aspect is devoted to the investigation of Ulam-Hyers type stability analysis which is also established. For the verification of our work, we provide some suitable examples along with their graphical represntation and errors estimates.
\end{abstract}

Keywords: Monotone iterative technique; Fractional differential equations; Extremal solutions; Ulam stability

\section{Introduction}

FDEs and their systems are the core purpose of most of the researchers because they have many important applications in various fields of advanced technology and science. There are many books and articles which have been written on the topic, see the works of Mainardi and Podlubny. Some applications where FDEs have been used are as follows:

- Modeling materials or systems which are dependent on past history (memory) such as nonlocal elasticity, biological tissues, propagation in complex medium, earth sediments, polymers, and expansion of the universe.

- Strong connection with fractal geometry.

- Can be derived even from probabilistic approaches.

Moreover, classical DEs are just discrete aspects of the whole spectrum of DEs, while FDEs give the total spectrum.

Although some phenomena have been produced in the form of integral order differential equations, in general, every phenomenon cannot be produced accurately in the form of integral order differential equations like the phenomena of electrochemistry, rheology, porous media, geology, electromagnetism, optics, medicine, bioscience, bioengineering, probability, and statistics. In this concern, most of the researchers prefer nonlinear FDEs, which are powerful tools, instead of the integral order differential equations for mathemat-

(c) The Author(s) 2019. This article is distributed under the terms of the Creative Commons Attribution 4.0 International License (http://creativecommons.org/licenses/by/4.0/), which permits unrestricted use, distribution, and reproduction in any medium, provided you give appropriate credit to the original author(s) and the source, provide a link to the Creative Commons license, and indicate if changes were made. 
ical modeling of the aforesaid phenomena. Therefore, plenty of the research items have been written on nonlinear FDEs, see [1-8] to explore more of their features for modeling purposes. Numerous properties and useful features of mathematical modeling of FDEs have been established by many authors in almost every field of science and technology such as ecology, control theory, seepage flow in porous media, and nonlinear oscillations due to earthquake (for details, see [9-15]). Furthermore, many researchers have devoted their works to the uniqueness and existence of solutions to FDEs; for reading, see [16-20]. As far as we know, the aforesaid area of fractional calculus has been greatly developed. The field of a coupled system of fractional differential equations is the main research field of most of the authors. It is the cascading form of single fractional differential equation. The handling of a coupled system of FDEs is a difficult task as compared with single fractional differential equation. In concern to the establishment of various aspects of the coupled system of FDEs is the need of science and technology because some phenomena describe more than one required task (solution) to be found. These phenomena can be expressed as a system of FDEs instead of DE/FDE. In this regard, some authors have devoted their treasured work to basic features and properties of the coupled systems of FDEs; for reading, see [21-24].

Existence and uniqueness of differential equations involving fractional derivatives have been considered in many works. For details on these works, we refer the readers to [2531 ] and the works cited therein. Recently, the existence and uniqueness of lower and upper solutions of FDEs have attracted the focus of most of researchers. Although some authors have well studied lower and upper solutions of integral order partial and ordinary differential equations using iterative techniques (see [32-42]), the iterative techniques have been rarely studied for the lower and upper solutions of FDEs as well as coupled systems of FDEs. It is a useful tool for the existence of approximation to the solutions of many applied problems of integral and differential equations of arbitrary order. The aforementioned scheme has been studied by some authors for FDEs. Quite recently, Ali [43] has considered this scheme (technique) for the following fractional differential equations:

$$
\left\{\begin{array}{l}
\mathbb{D}^{\alpha} \theta(\xi)+f(\xi, \theta(\xi))=0, \quad \xi \in(0,1), 1<\alpha<2 \\
\theta^{\prime}(0)=0, \quad \theta(1)=\xi \theta(\varrho)
\end{array}\right.
$$

where $\xi, \varrho \in(0,1)$.

As far as we know, the aforementioned scheme for the coupled system of FDEs is in its initial stage.

On the other hand, stability aspect is the most popular topic in the discussion among most of the researchers. It has been well studied in major research articles for the classical differential equations. Some types of stability analysis for both classical differential equations and FDEs, like Lyapunov, exponential and Ulam-Hyers type stability, etc., have been developed. Among them, Ulam-Hyers type stability is fundamental and very important stability analysis. It has been verified in many articles for the classical differential equations as well as fractional differential equations. Ulam-Hyers stability analysis has been well studied for the initial value problems of fractional differential equations. As far as we know, it has been rarely investigated for the boundary value problems of fractional differential equations; for reading, see [44-48]. 
The aim of our manuscript is to extend the scheme of Ali [43] to coupled systems of FDEs. We consider the following class of nonlinear fractional coupled system of higher order boundary conditions:

$$
\left\{\begin{array}{l}
\mathbb{D}^{p} \theta(\xi)+\Phi(\xi, \vartheta(\xi))=0, \quad \xi \in[0,1], \\
\mathbb{D}^{q} \vartheta(\xi)+\Psi(\xi, \theta(\xi))=0, \quad \xi \in[0,1], \\
\theta(1)=\theta^{\prime}(0)=\theta^{\prime \prime}(0)=\cdots=\theta^{(n-2)}(0)=\theta^{(n-1)}(0)=0, \\
\vartheta(1)=\vartheta^{\prime}(0)=\vartheta^{\prime \prime}(0)=\cdots=\vartheta^{(n-2)}(0)=\vartheta^{(n-1)}(0)=0,
\end{array}\right.
$$

where $n=2,3,4, \ldots, n-1<q, p \leq n, \theta, y \in C[0,1]$.

$\mathbb{D}$ is considered in the sense of Caputo derivative assuming that $\Phi, \Psi: I \times \mathbb{R} \rightarrow \mathbb{R}$.

This manuscript has the following main contributions:

- To extend and develop the iterative scheme while combining the method of lower and upper solutions for the nonlinear fractional coupled system of higher order boundary conditions.

- Existence of lower and upper solutions of the nonlinear fractional coupled system of higher order boundary conditions.

- The investigation of Ulam-Hyers stability for the coupled system of nonlinear FDEs of higher order boundary conditions.

- Approximations of extremal solutions of the nonlinear fractional coupled system of higher order boundary conditions.

- Provision of maximum errors estimate of extremal solutions of the nonlinear fractional coupled system of higher order boundary conditions.

- The presentation of lower and upper solutions of the nonlinear fractional coupled system of higher order boundary conditions via plots using Matlab software.

Three illustrative examples are provided to demonstrate the obtained results. Further we remark that Caputo type fractional order derivative is considered throughout this paper.

\section{Background materials}

In the present section of our work, we mention supportive basic definitions with useful lemmas from fractional calculus as well as measure theory and functional analysis; for reading, see $[2-5,19,20,37]$.

Definition 2.1 Let $\theta(\xi) \in L([0,1], \mathbb{R})$ be a function. Then the Riemann-Liouville integral of fractional order $\delta \in \mathbb{R}_{+}$of function $\theta(\xi)$ is given by

$$
I^{\delta} \theta(\xi)=\frac{1}{\Gamma(\delta)} \int_{0}^{\xi}(\xi-\sigma)^{\delta-1} \theta(\sigma) d \sigma,
$$

provided that the integral is pointwise defined on the right-hand side.

Definition 2.2 ([3]) The Caputo derivative of fractional order $p$ of function $\theta(\xi)$ is defined by

$$
\mathbb{D}^{p} \theta(\xi)=\frac{1}{\Gamma(n-p)} \int_{0}^{\xi}(\xi-\sigma)^{n-p-1} \theta^{(n)}(\sigma) d \sigma
$$


provided that the integral on the right-hand side is pointwise defined on $(0, \infty)$, where $n=[p]+1$ and $[p]$ denotes the integer part of the real number $p$.

Definition 2.3 As in $[21,43]$, "Let $\mathcal{U}=C[0,1]$ be the Banach space endowed with $\|\theta\|=$ $\max _{\xi \in[0,1]}|\theta(\xi)|$ which satisfies the partial ordering, and let $W=\left[\theta_{1}, \theta_{2}\right]$ with $\theta_{1} \leq \theta_{2}$ be a set such that $W \subset \mathcal{U}$, then the operator $\mathbb{T}: W \rightarrow \mathcal{U}$ is known as increasing function if for each $\theta_{1}, \theta_{2} \in W$ and $\theta_{1} \leq \theta_{2}$ gives $\mathbb{T} \theta_{1} \leq \mathbb{T} \theta_{2}$. The operator $\mathbb{T}$ is known as decreasing function if for each $\theta_{1}, \theta_{2} \in W$ gives $\mathbb{T} \theta_{1} \geq \mathbb{T} \theta_{2}$."

Definition 2.4 As in [21, 43], "Let $I$ be an identity operator. If $(I-\mathbb{T}) \theta_{1} \leq 0$, then the function $\theta_{1} \in W$ is known as a minimal solution of $(I-\mathbb{T}) \theta=0$ and if $(I-\mathbb{T}) \theta_{2} \geq 0$, then the function $\theta_{2} \in W$ is known as a maximal solution of $(I-\mathbb{T}) \theta=0$."

Lemma 2.5 ([20]) For the Banach space $\mathcal{U}$ which partially satisfies order with $W \subset \mathcal{U}$ and $\theta_{n}, \theta_{n}^{*} \in W$ such that $\theta_{n} \leq \theta_{n}^{*}, n \in Z^{+}$. If $\theta_{n} \rightarrow \theta$ and $\theta_{n}^{*} \rightarrow \theta^{*}$, then $\theta \leq \theta^{*}$.

Lemma $2.6([3,49])$ Let $p>0$, then the FDE

$$
\mathbb{D}^{p} \theta(\xi)=0
$$

has the solution in the form of

$$
\theta(\xi)=\sum_{i=0}^{[p]} \frac{\theta^{(i)}(0)}{i !} t^{i}
$$

Lemma $2.7([3,49])$ Let $p>0$, then

$$
I^{p}\left[\mathbb{D}^{p} \theta(\xi)\right]=\theta(\xi)-\sum_{i=0}^{[p]} \frac{\theta^{(i)}(0)}{i !} t^{i} .
$$

\section{Iterative solutions}

This section is committed to the existence theory, approximation and error estimates to the extremal solution of system (1). In the first attempt we transfer system (1) to the equivalent system of integral equations. It is to be noted that the integral representation of the coupled system is very useful for onward computations. Green's function in the integral representation is also necessary to be computed for iterative solution. The next step after the integral representation is establishing sufficient conditions for the existence of extremal solutions to system (1). We also provide iterative approximation to extremal solution of the system by a monotone iterative scheme coupled with the lower and upper solutions method. Further, by the convergent property of monotonic sequence, we consequently compute the error estimates for the iterative solution to extremal solutions of system (1). For the aforesaid concerns and computations, the following assumptions are considered:

$\left(C_{1}\right)$ The real-valued functions $\Phi, \Psi:[0,1] \times \mathbb{R} \rightarrow \mathbb{R}$ satisfy the Carathéodory lemma (for reading, see the preliminary section of [20]).

$\left(C_{2}\right)$ The functions $\Phi(\xi, \theta)$ and $\Psi(\xi, \vartheta)$ are increasing in $\theta$ and $\vartheta$ for every $\xi \in[0,1]$ respectively. 
$\left(C_{3}\right)$ The existence of the constants $\mathbb{A}, \mathbb{B}>0$ such that $0 \leq \Phi\left(\xi, \theta_{1}(\xi)\right)-\Phi\left(\xi, \theta_{2}(\xi) \leq\right.$ $\mathbb{A}\left(\theta_{1}-\theta_{2}\right)$ and $0 \leq \Psi\left(\xi, \vartheta_{1}(\xi)\right)-\Psi\left(\xi, \vartheta_{2}(\xi) \leq \mathbb{B}\left(\vartheta_{1}-\vartheta_{2}\right)\right.$.

Lemma 3.1 In view of $C_{1}$ with $h \in C([0,1], \mathbb{R})$, then the fractional differential equation

$$
\begin{aligned}
& \mathbb{D}^{p} \theta(\xi)+h(\xi)=0, \quad \xi \in[0,1],(n-1)<p \leq n, \\
& \theta(1)=\theta^{\prime}(0)=\theta^{\prime \prime}(0)=\cdots=\theta^{(n-2)}(0)=\theta^{(n-1)}(0)=0
\end{aligned}
$$

has the solution defined by

$$
\theta(\xi)=\int_{0}^{1} \mathbb{G}_{1}(\xi, \sigma) h(\sigma) d \sigma
$$

where $\mathbb{G}_{1}(\xi, \sigma)$ is given by

$$
\mathbb{G}_{1}(\xi, \sigma)=\frac{1}{\Gamma(p)} \begin{cases}(1-\sigma)^{p-1}-(\xi-\sigma)^{p-1}, & 0 \leq \sigma \leq \xi \leq 1 \\ (1-\sigma)^{p-1}, & 0 \leq \xi \leq \sigma \leq 1\end{cases}
$$

$\mathbb{G}_{1}(\xi, \sigma)$ is known as Green's function.

Proof In view of Lemma 2.7 and derivative considered in the sense of Caputo derivative, the linear BVP (2) yields that

$$
\theta(\xi)=C_{1}+C_{2} \xi+\cdots+C_{n} \xi^{n-1}-I^{p} h(\xi) .
$$

We face singularity by using the conditions $\theta^{\prime}(0)=\theta^{\prime \prime}(0)=\cdots=\theta^{(n-2)}(0)=\theta^{(n-1)}(0)=0$. Therefore to avoid singularity, here we have $C_{2}=C_{3}=C_{4}=\cdots=C_{n}=0$. Further, as $\theta(1)=$ 0 , then we have $C_{1}=I^{p} h(1)$. Therefore, we deduce from (5) that

$$
\theta(\xi)=\int_{0}^{1} \frac{(1-\sigma)^{p-1}}{\Gamma(p)} h(\sigma) d \sigma-\int_{0}^{\xi} \frac{(\xi-\sigma)^{p-1}}{\Gamma(p)} h(\sigma) d \sigma .
$$

Hence, we obtain the result from (6) that is

$$
\theta(\xi)=\int_{0}^{1} \mathbb{G}_{1}(\xi, \sigma) h(\sigma) d \sigma .
$$

This is the end of the proof.

Lemma 3.2 Let $W=C[0,1]$ be the Banach space with $\|\theta\|=\max _{\xi \in[0,1]}|\theta(\xi)|$. Then the coupled system (1) has the integral representation

$$
\begin{cases}\theta(\xi)=\int_{0}^{1} \mathbb{G}_{1}(\xi, \sigma) \Phi(\sigma, \vartheta(\sigma)) d \sigma, & \xi \in[0,1] \\ \vartheta(\xi)=\int_{0}^{1} \mathbb{G}_{2}(\xi, \sigma) \Psi(\sigma, \theta(\sigma)) d \sigma, & \xi \in[0,1]\end{cases}
$$

where $\mathbb{G}_{1}(\xi, \sigma)$ is given in (4) and

$$
\mathbb{G}_{2}(\xi, \sigma)= \begin{cases}\frac{(1-\sigma)^{q-1}-(\xi-\sigma)^{q-1}}{\Gamma(q)}, & 0 \leq \sigma \leq \xi \leq 1 \\ \frac{(1-\sigma)^{q-1}}{\Gamma(q)}, & 0 \leq \xi \leq \sigma \leq 1\end{cases}
$$


Clearly, $\mathbb{G}_{1}(\xi, \sigma) \geq 0, \mathbb{G}_{2}(\xi, \sigma) \geq 0$ for all $\xi, \sigma \in[0,1]$.

Lemma 3.3 The functions $\mathbb{G}_{1}, \mathbb{G}_{2}$ satisfy the following property:

$$
\int_{0}^{1}\left|\mathbb{G}_{1}(\xi, \sigma)\right| d \sigma \leq \frac{1}{\Gamma(p+1)}, \quad \int_{0}^{1}\left|\mathbb{G}_{2}(\xi, \sigma)\right| d \sigma \leq \frac{1}{\Gamma(q+1)} \quad \text { for all } \xi \in[0,1]
$$

Proof As

$$
\frac{(1-\sigma)^{p-1}-(\xi-\sigma)^{p-1}}{\Gamma(p)} \leq \frac{(1-\sigma)^{p-1}}{\Gamma(p)}
$$

implies that

$$
\mathbb{G}_{1}(\xi, \sigma) \leq \frac{(1-\sigma)^{p-1}}{\Gamma(p)}
$$

thus

$$
\left|\mathbb{G}_{1}(\xi, \sigma)\right| \leq \frac{(1-\sigma)^{p-1}}{\Gamma(p)}
$$

from which we have

$$
\int_{0}^{1}\left|\mathbb{G}_{1}(\xi, \sigma)\right| d \sigma \leq \frac{1}{\Gamma(p+1)}
$$

Similarly, we can prove that $\int_{0}^{1}\left|\mathbb{G}_{2}(\xi, \sigma)\right| d \sigma \leq \frac{1}{\Gamma(q+1)}$.

Hence, the proof is completed.

We write the coupled system (7) of integral equations as

$$
\begin{aligned}
\theta(\xi) & =\int_{0}^{1} \mathbb{G}_{1}(\xi, \sigma) \Phi(\sigma, \vartheta(\sigma)) d \sigma \\
& =\int_{0}^{1} \mathbb{G}_{1}(\xi, \sigma) \Phi\left(\sigma, \int_{0}^{1} \mathbb{G}_{2}(\sigma, \varrho) \Psi(\varrho, \theta(\varrho)) d \varrho\right) d \sigma
\end{aligned}
$$

Further, we define $\mathbb{T}: W \rightarrow \mathcal{U}$ is an operator by

$$
\mathbb{T}(\theta(\xi))=\int_{0}^{1} \mathbb{G}_{1}(\xi, \sigma) \Phi\left(\sigma, \int_{0}^{1} \mathbb{G}_{2}(\sigma, \varrho) \Psi(\varrho, \theta(\varrho)) d \varrho\right) d \sigma .
$$

Thanks to equation (8) and equation (9), we obtain the equation

$$
[I-\mathbb{T}(\theta(\xi))]=0, \quad \xi \in[0,1]
$$


It is to be noted that equations (8) and (10) have the same solutions that are fixed points of $\mathbb{T}$. As in $C_{2}$, we have that, for $\theta, \theta^{*} \in W$ with $\theta \leq \theta^{*}$, we obtain

$$
\begin{aligned}
\mathbb{T}(\theta(\xi)) & =\int_{0}^{1} \mathbb{G}_{1}(\xi, \sigma) \Phi\left(\sigma, \int_{0}^{1} \mathbb{G}_{2}(\sigma, \varrho) \Psi(\varrho, \theta(\varrho)) d \varrho\right) d \sigma \\
& \leq \int_{0}^{1} \mathbb{G}_{1}(\xi, \sigma) \Phi\left(\sigma, \int_{0}^{1} \mathbb{G}_{2}(\sigma, \varrho) \Psi\left(\varrho, \theta^{*}(\varrho)\right) d \varrho\right) d \sigma=\mathbb{T}\left(\theta^{*}(\xi)\right)
\end{aligned}
$$

implies that

$$
\mathbb{T}(\theta(\xi)) \leq \mathbb{T}\left(\theta^{*}(\xi)\right)
$$

thus $\mathbb{T}$ is an increasing operator.

$\left(C_{4}\right)$ Assume that the lower and upper solutions of (10) are $\alpha$ and $\beta \in W$. Then the inequality $\alpha \leq \beta$ on $[0,1]$ holds.

Lemma 3.4 Consider all the assumptions $\left(C_{1}\right)$ to $\left(C_{4}\right)$. Then the solutions of linear problems are an iterative convergent sequence which converges to solution of the integral equation (8).

Proof Under assumptions $\left(C_{1}\right),\left(C_{2}\right)$, and $\left(C_{4}\right)$, let $\theta_{i}, \theta_{j} \in W \subset \mathcal{U}$, where $i, j=1,2,3, \ldots, n$

$$
\begin{aligned}
\mid \mathbb{T} & \left(\theta_{i}(\xi)\right)-\mathbb{T}\left(\theta_{j}(\xi)\right) \mid \\
= & \mid \int_{0}^{1} \mathbb{G}_{1}(\xi, \sigma) \Phi\left(\sigma, \int_{0}^{1} \mathbb{G}_{2}(\sigma, \varrho) \Psi\left(\varrho, \theta_{i}(\varrho)\right) d \varrho\right) d \sigma \\
& \quad-\int_{0}^{1} \mathbb{G}_{1}(\xi, \sigma) \Phi\left(\sigma, \int_{0}^{1} \mathbb{G}_{2}(\sigma, \varrho) \Psi\left(\varrho, \theta_{j}(\varrho)\right) d \varrho\right) d \sigma \mid \\
\leq & \int_{0}^{1} \mathbb{G}_{1}(\xi, \sigma) \mid \Phi\left(\sigma, \int_{0}^{1} \mathbb{G}_{2}(\sigma, \varrho) \Psi\left(\varrho, \theta_{i}(\varrho)\right) d \varrho\right) \\
& -\Phi\left(\sigma, \int_{0}^{1} \mathbb{G}_{2}(\sigma, \varrho) \Psi\left(\varrho, \theta_{j}(\varrho)\right) d \varrho\right) \mid d \sigma \\
\leq & \int_{0}^{1} \mathbb{G}_{1}(\xi, \sigma) \mathbb{A} \int_{0}^{1} \mathbb{G}_{2}(\sigma, \varrho)\left|\Psi\left(\varrho, \theta_{i}(\varrho)\right)-\Psi\left(\varrho, \theta_{j}(\varrho)\right)\right| d \varrho d \sigma \\
\leq & \frac{\mathbb{A} \mathbb{B}}{\Gamma(q+1)} \Gamma(p+1)\left\|\theta_{i}-\theta_{j}\right\|=\nabla\left\|\theta_{i}-\theta_{j}\right\|, \quad \text { where } \nabla=\frac{\mathbb{A B}}{\Gamma(q+1)} \Gamma(p+1) .
\end{aligned}
$$

Therefore, the operator $\mathbb{T}$ is continuous. Further, it is obvious that $\mathbb{T}$ is also equicontinuous and uniformly bounded. Arzelá-Ascoli theorem which states that "If $M$ is a family (finite or infinite) of an equi-continuous, uniformly bounded real-valued functions $\theta$ on an interval $[0,1]$, then $M$ contains a uniformly convergent sequence of functions $\theta_{n}$, converging to a function $\theta$ in $W$ as $n \rightarrow \infty$, where $W$ denotes the space of all continuous bounded functions on $[0,1]$. Thus any sequence in $M$ contains a uniformly bounded convergent subsequence on $[0,1]$ and consequently $M$ has a compact closure in $W$." Hence, in view of the Arzelá-Ascoli theorem, $\mathbb{T}$ is also a compact operator. Let the lower solution of integral equation (10) be $\theta_{0}=\alpha$. Then, in view of condition $\left(C_{4}\right)$, we obtain

$$
\theta_{0} \leq \beta
$$


As $\mathbb{T}$ is an increasing operator, then we obtain

$$
\theta_{0} \leq \mathbb{T} \theta_{0} \leq \mathbb{T} \beta \leq \beta, \quad \text { that is, } \theta_{0} \leq \theta_{1} \leq \beta \text { on the interval }[0,1]
$$

where $\theta_{1}=\mathbb{T} \theta_{0}$ is an iterative solution of integral equation (10). By applying $\mathbb{T}$, we obtain

$$
\mathbb{T} \theta_{0} \leq \mathbb{T} \theta_{1} \leq \mathbb{T} \beta \leq \beta, \quad \text { that is, } \theta_{1} \leq \theta_{2} \leq \beta \text { on }[0,1]
$$

where $\theta_{2}=\mathbb{T} \theta_{1}$. Consequently, we obtain a bounded monotone sequence $\left\{\theta_{n}\right\}$ which is

$$
\theta_{0} \leq \theta_{1} \leq \theta_{2} \leq \cdots \leq \theta_{n-1} \leq \theta_{n} \leq \beta \quad \text { on }[0,1]
$$

where $\theta_{n}=\mathbb{T} \theta_{n-1}$ is solution of equation (10). Thus, in view of the bounded monotone sequence $\left\{\theta_{n}\right\}$, there exists $\theta \in W$ so that $\theta_{n} \rightarrow \theta$ as $n \rightarrow \infty$. Therefore $\theta=\mathbb{T} \theta$, which is the solution of integral equation (8) defined by

$$
\theta(\xi)=\int_{0}^{1} \mathbb{G}_{1}(\xi, \sigma) \Phi\left(\sigma, \int_{0}^{1} \mathbb{G}_{2}(\sigma, \varrho) \Psi(\varrho, \theta(\varrho)) d \varrho\right) d \sigma, \quad \xi \in[0,1]
$$

Hence, in view of (13) and (12), we get

$$
\begin{aligned}
& \left\|\theta_{2}-\theta_{1}\right\|=\left\|\mathbb{T} \theta_{1}-\mathbb{T} \theta_{0}\right\| \leq \nabla e_{1}, \\
& \left\|\theta_{3}-\theta_{2}\right\|=\left\|\mathbb{T} \theta_{2}-\mathbb{T} \theta_{1}\right\| \leq \nabla^{2} e_{1}, \\
& \left\|\theta_{4}-\theta_{3}\right\|=\left\|\mathbb{T} \theta_{3}-\mathbb{T} \theta_{2}\right\| \leq \nabla^{3} e_{1}, \\
& \vdots \\
& \left\|\theta_{n+1}-\theta_{n}\right\|=\left\|\mathbb{T} \theta_{n}-\mathbb{T} \theta_{n-1}\right\| \leq \nabla^{n} e_{1} .
\end{aligned}
$$

Consequently, for positive integers $m$ and $n$, we have

$$
\begin{aligned}
\left\|\theta_{m+n}-\theta_{n}\right\| & \leq\left\|\theta_{n+m}-\theta_{n+m-1}\right\|+\left\|\theta_{n+m-1}-\theta_{n+m-2}\right\|+\cdots+\left\|\theta_{n+1}-\theta_{n}\right\| \\
& \leq \nabla^{n} \frac{1-\nabla^{m}}{1-\nabla} e_{1} .
\end{aligned}
$$

It is obvious that $\nabla<1$, which implies that $\left\|\theta_{m+n}-\theta_{n}\right\| \rightarrow 0$ when $n \rightarrow \infty$. Thus $\theta_{n}$ is a Cauchy sequence in $W$. Let $\theta^{*}(\xi)=\lim _{n \rightarrow \infty} \theta_{n}(\xi)$, thus $\mathbb{T} \theta^{*}=\theta^{*}$. Therefore, if $m \rightarrow \infty$ in (14), then error estimate for the lower solution is

$$
e_{n}=\left\|\theta^{*}-\theta_{n}\right\| \leq \frac{\nabla^{n}}{1-\nabla} e_{1}, \quad \text { where } e_{1}=\left\|\theta_{1}-\theta_{0}\right\|
$$

Remark Let us choose $\theta_{0}=\beta$, we have a sequence $\left\{\theta_{n}\right\}$ so that

$$
\theta_{0} \geq \theta_{1} \geq \theta_{2} \geq \cdots \geq \theta_{n-1} \geq \theta_{n} \geq \alpha \quad \text { on }[0,1]
$$

which converges to a solution of the integral equation (8). Therefore, we can obtain the estimate error for the maximal solution which is given by $e_{n}^{*}=\left\|\theta_{n}^{*}-\bar{\theta}^{*}\right\| \leq \frac{\nabla^{n}}{1-\nabla} e_{1}^{*}$, 
where $e_{1}^{*}=\left\|\theta_{0}^{*}-\theta_{1}^{*}\right\|$. Further, we use Lemma 3.4, the iterative sequences for lower and upper solutions of system (1) are:

$$
\begin{aligned}
& \theta_{n}(\xi)=\int_{0}^{1} \mathbb{G}_{1}(\xi, \sigma) \Phi\left(\sigma, \int_{0}^{1} \mathbb{G}_{2}(\sigma, \varrho) \Psi\left(\varrho, \theta_{n-1}(\varrho)\right) d \varrho\right) d \sigma, \quad n \geq 1 \\
& \theta_{n}^{*}(\xi)=\int_{0}^{1} \mathbb{G}_{1}(\xi, \sigma) \Phi\left(\sigma, \int_{0}^{1} \mathbb{G}_{2}(\sigma, \varrho) \Psi\left(\varrho, \theta_{n-1}^{*}(\varrho)\right) d \varrho\right) d \sigma, \quad n \geq 1 .
\end{aligned}
$$

Hence, we have

$$
\begin{aligned}
& \theta^{*}(\xi)=\lim _{n \rightarrow \infty} \theta_{n}(\xi), \vartheta^{*}(\xi)=\int_{0}^{1} \mathbb{G}_{2}(\xi, \sigma) \Psi\left(\sigma, \theta^{*}(\sigma)\right) d \sigma, \\
& \bar{\theta}^{*}(\xi)=\lim _{n \rightarrow \infty} \theta_{n}^{*}(\xi), \bar{\vartheta}^{*}(\xi)=\int_{0}^{1} \mathbb{G}_{2}(\xi, \sigma) \Psi\left(\sigma, \bar{\theta}^{*}(\sigma)\right) d \sigma .
\end{aligned}
$$

Theorem 3.5 Consider conditions $\left(C_{1}\right),\left(C_{2}\right)$, and $\left(C_{3}\right)$ and $\nabla<1$. Then the coupled system of BVPs (1) has unique minimal and maximal solutions respectively.

Proof We prove that $\left(\theta^{*}, \vartheta^{*}\right)$ and $\left(\bar{\theta}^{*}, \vartheta^{*}\right)$ are the minimal and maximal solutions of (7). Let for any $u(\xi) \in W$ with $\mathbb{T} u=u$ and $\theta_{n} \leq u \leq \theta_{n}^{*}$, due to the increasing behavior of $\mathbb{T}$ and using Lemma 3.4, we have $\theta^{*}(\xi) \leq u(\xi) \leq \theta^{*}(\xi)$ that $\theta^{*}(\xi)$ and $\theta^{*}(\xi)$ are the minimal and maximal fixed points of $\mathbb{T}$ respectively. Therefore $\left(\theta^{*}, \vartheta^{*}\right)$ and $\left(\bar{\theta}^{*}, \bar{\vartheta}^{*}\right)$ are the minimal and maximal solution of (7) respectively.

Next, for the concern of uniqueness of minimal and maximal solutions of system (7), let $\alpha, \beta \in W$ be the lower and upper solutions of $\mathbb{T} \theta=\theta$ respectively. Then $\alpha \leq \mathbb{T} \alpha, \beta \geq \mathbb{T} \beta$, $\xi \in[0,1]$. We use $\alpha$ and $\beta$ as initial iterations respectively so that $\alpha_{n} \rightarrow \alpha^{*}$ and $\beta_{n} \rightarrow \beta^{*}$, $n \rightarrow \infty$. We also have $\mathbb{T} \alpha^{*}=\alpha^{*}, \mathbb{T} \beta^{*}=\beta^{*}$. To prove $\alpha^{*}=\theta^{*}$, observe that $\theta_{0} \leq \alpha^{*}$ and $\mathbb{T}$ is increasing, so we have $\theta_{n}=\mathbb{T}^{n} \theta_{0} \leq \mathbb{T}^{n} \alpha^{*}$ for each $n \in\{1,2,3, \ldots\}$. Then $\theta_{0} \leq \theta_{1} \leq$ $\theta_{2} \leq \cdots \leq \theta_{n} \leq \cdots \leq \alpha^{*}$. Therefore from (12) and mathematical induction, it is obvious that $\left\|\alpha^{*}-\theta_{n}\right\|=\left\|\mathbb{T}^{n} \alpha^{*}-\mathbb{T}^{n} \theta_{0}\right\| \leq \nabla^{n}\left\|\alpha^{*}-\theta_{0}\right\| \rightarrow 0$ as $n \rightarrow \infty$. Therefore $\left\|\alpha^{*}-\theta^{*}\right\| \rightarrow 0$ as $n \rightarrow \infty$, which gives $\theta^{*}=\alpha^{*}$. In the same manner, we obtain that $\bar{\theta}^{*}=\beta^{*}$. Thus, the minimal and maximal solutions of the coupled system (7) are unique.

\section{Generalized Ulam-Hyers stability of the solutions of BVPs (1)}

This section is devoted to the investigation of more general stability analysis for the system of fractional differential equations. The stability analysis is an important aspect of fractional calculus for the ordinary and partial differential equations of fractional order. As far as we know, it is in the initial stage for a coupled system. Therefore the aim of this section is to investigate some sufficient conditions for a system of fractional differential equations. In this concern, we study Ulam-Hyers and generalized Ulam-Hyers stability for a coupled system of BVPs (1) of the nonlinear FDEs. For this concern, we require the following auxiliary definitions.

Definition 4.1 The considered problem (1) is Ulam-Hyers stable if we can find a real number $\hat{C}_{\Phi, \Psi}>0$ with the property that, for every $\varepsilon=\max \left\{\varepsilon_{1}, \varepsilon_{2}\right\}>0$ with $\varepsilon_{1}>0$ and 
$\varepsilon_{2}>0$ and for every solution $(\theta, \vartheta) \in C([0,1], \mathbf{R}) \times C([0,1], \mathbf{R})$ of the inequality

$$
\begin{aligned}
& \left|\mathbb{D}^{p} \theta(\xi)-\Phi(\xi, \vartheta(\xi))\right| \leq \varepsilon_{1}, \quad \xi \in[0,1], \\
& \left|\mathbb{D}^{q} \vartheta(\xi)-\Psi(\xi, \theta(\xi))\right| \leq \varepsilon_{2}, \quad \xi \in[0,1],
\end{aligned}
$$

there exists a unique solution $(\tilde{\theta}, \tilde{\vartheta}) \in C([0,1], \mathbf{R}) \times C([0,1], \mathbf{R})$ of the proposed BVPs $(1)$ with

$$
\|(\theta, \vartheta)-(\tilde{\theta}, \tilde{\vartheta})\| \leq \hat{C}_{\Phi, \Psi} \varepsilon, \quad \xi \in[0,1]
$$

Definition 4.2 The proposed BVPs (1) is called to be generalized Ulam-Hyers stable if we can find $\Theta_{\Phi, \Psi}:(0, \infty) \rightarrow \mathbf{R}^{+}$with $\Theta_{\Phi, \Psi}(0)=0$, with the property that, for every $\varepsilon=\max \left\{\varepsilon_{1}, \varepsilon_{2}\right\}>0$ with $\varepsilon_{1}>0$ and $\varepsilon_{2}>0$ and for every solution $(\theta, \vartheta) \in C([0,1], \mathbf{R}) \times$ $C([0,1], \mathbf{R})$ of the inequality

$$
\begin{aligned}
& \left|\mathbb{D}^{p} \theta(\xi)-\Phi(\xi, \vartheta(\xi))\right| \leq \varepsilon_{1}, \quad \xi \in[0,1], \\
& \left|\mathbb{D}^{q} \vartheta(\xi)-\Psi(\xi, \theta(\xi))\right| \leq \varepsilon_{2}, \quad \xi \in[0,1],
\end{aligned}
$$

there exists a unique solution $(\tilde{\theta}, \tilde{\vartheta}) \in C([0,1], \mathbf{R}) \times C([0,1], \mathbf{R})$ of the proposed BVPs (1) with

$$
\|(\theta, \vartheta)-(\tilde{\theta}, \tilde{\vartheta})\| \leq \hat{C}_{\Phi, \Psi} \Theta_{\Phi, \Psi}(\xi), \quad \xi \in[0,1] .
$$

Remark 4.3 The $(\theta, \vartheta) \in C([0,1], \mathbb{R})$ is said to be the solution of BVPs (1) and satisfy the inequality given in (15) if and only if we can find a function $\alpha, \beta \in C([0,1], \mathbb{R})$ depending only on $(\theta, \vartheta)$ respectively, then

(i) $|\alpha(\xi)| \leq \varepsilon_{1},|\beta(\xi)| \leq \varepsilon_{2}$, for all $\xi \in[0,1]$;

(ii) $\mathbb{D}^{p} \theta(\xi)+\Phi(\xi, \vartheta(\xi))=\alpha(\xi)$, for all $\xi \in[0,1], \mathbb{D}^{q} \vartheta(\xi)+\Psi(\xi, \theta(\xi))=\beta(\xi)$, for all $\xi \in[0,1]$.

Thanks to Remark 4.3 and assumption $\left(C_{3}\right)$ for $\xi \in([0,1])$, the considered solution $(\theta, \vartheta)$ of the problem

$$
\left\{\begin{array}{l}
\mathbb{D}^{p} \theta(\xi)+\Phi(\xi, \vartheta(\xi))=\alpha(\xi), \quad \xi \in[0,1], \\
\mathbb{D}^{q} \vartheta(\xi)+\Psi(\xi, \theta(\xi))=\beta(\xi), \quad \xi \in[0,1], \\
\theta(1)=\theta^{\prime}(0)=\theta^{\prime \prime}(0)=\cdots=\theta^{(n-2)}(0)=\theta^{(n-1)}(0)=0, \\
\vartheta(1)=\vartheta^{\prime}(0)=\vartheta^{\prime \prime}(0)=\cdots=\vartheta^{(n-2)}(0)=\vartheta^{(n-1)}(0)=0,
\end{array}\right.
$$


given by

$$
\begin{aligned}
\theta(\xi)= & \int_{0}^{1} \mathbb{G}_{1}(\xi, \sigma) \Phi\left(\sigma, \int_{0}^{1} \mathbb{G}_{2}(\xi, \varrho) \Psi(\varrho, \theta(\varrho)) d \varrho+\int_{0}^{1} K_{2}(\xi, \varrho) \beta(\varrho) d \varrho\right) d \sigma \\
& +\int_{0}^{1} \mathbb{G}_{1}(\xi, \sigma) \alpha(\sigma) d \sigma, \quad \xi \in[0,1], \\
\vartheta(\xi)= & \int_{0}^{1} \mathbb{G}_{2}(\xi, \sigma) \Psi\left(\sigma, \int_{0}^{1} \mathbb{G}_{1}(\xi, \varrho) \Phi(\varrho, \vartheta(\varrho)) d \varrho+\int_{0}^{1} K_{1}(\xi, \varrho) \alpha(\varrho) d \varrho\right) d \sigma \\
& +\int_{0}^{1} \mathbb{G}_{2}(\xi, \sigma) \beta(\sigma) d \sigma, \quad \xi \in[0,1],
\end{aligned}
$$

satisfies the following inequality:

$$
\begin{aligned}
& \left|\theta(\xi)-\int_{0}^{1} \mathbb{G}_{1}(\xi, \sigma) \Phi\left(\sigma, \int_{0}^{1} \mathbb{G}_{2}(\xi, \varrho) \Psi(\varrho, \theta(\varrho)) d \varrho+\int_{0}^{1} K_{2}(\xi, \varrho) \beta(\varrho) d \varrho\right) d \sigma\right| \\
& \quad \leq \frac{\varepsilon_{2}}{\Gamma(p+1)}, \quad \xi \in[0,1]
\end{aligned}
$$

and

$$
\begin{aligned}
& \left|\vartheta(\xi)-\int_{0}^{1} \mathbb{G}_{2}(\xi, \sigma) \Psi\left(\sigma, \int_{0}^{1} \mathbb{G}_{1}(\xi, \varrho) \Phi(\varrho, \vartheta(\varrho)) d \varrho+\int_{0}^{1} K_{1}(\xi, \varrho) \alpha(\varrho) d \varrho\right) d \sigma\right| \\
& \quad \leq \frac{\varepsilon_{1}}{\Gamma(q+1)}
\end{aligned}
$$

Theorem 4.4 Let $\nabla<1$ and assumption $\left(C_{3}\right)$ hold. Then solutions of the considered coupled system (1) are Ulam-Hyers stable and consequently generalized Ulam-Hyers stable.

Proof Let $(\theta, \vartheta)$ be any solution of BVPs (1)and satisfy inequality (15), and let $(\bar{\theta}, \bar{\vartheta})$ be the unique solution of the considered BVPs problem, then consider

$$
\begin{aligned}
|\theta-\bar{\theta}| & \\
= & \left|\theta-\int_{0}^{1} \mathbb{G}_{1}(\xi, \sigma) \Phi\left(\sigma, \int_{0}^{1} \mathbb{G}_{2}(\xi, \varrho) \Psi(\varrho, \bar{\theta}(\varrho)) d \varrho+\int_{0}^{1} K_{2}(\xi, \varrho) \beta(\varrho) d \varrho\right) d \sigma\right| \\
\leq & \left|\theta-\int_{0}^{1} \mathbb{G}_{1}(\xi, \sigma) \Phi\left(\sigma, \int_{0}^{1} \mathbb{G}_{2}(\xi, \varrho) \Psi(\varrho, \theta(\varrho)) d \varrho+\int_{0}^{1} K_{2}(\xi, \varrho) \beta(\varrho) d \varrho\right) d \sigma\right| \\
& +\mid \int_{0}^{1} \mathbb{G}_{1}(\xi, \sigma) \Phi\left(\sigma, \int_{0}^{1} \mathbb{G}_{2}(\xi, \varrho) \Psi(\varrho, \theta(\varrho)) d \varrho+\int_{0}^{1} K_{2}(\xi, \varrho) \beta(\varrho) d \varrho\right) d \sigma \\
& -\int_{0}^{1} \mathbb{G}_{1}(\xi, \sigma) \Phi\left(\sigma, \int_{0}^{1} \mathbb{G}_{2}(\xi, \varrho) \Psi(\varrho, \bar{\theta}(\varrho)) d \varrho+\int_{0}^{1} K_{2}(\xi, \varrho) \beta(\varrho) d \varrho\right) d \sigma \mid .
\end{aligned}
$$

Therefore from inequality (19)

$$
\begin{aligned}
& \|\theta-\bar{\theta}\| \leq \frac{\varepsilon_{2}}{\Gamma(p+1)}+\nabla\|\theta-\bar{\theta}\|, \\
& \|\theta-\bar{\theta}\| \leq \varepsilon_{2} \hat{C}_{\Phi, \Psi}, \quad \hat{C}_{\Phi, \Psi}=\frac{1}{(1-\nabla) \Gamma(p+1)}>0 .
\end{aligned}
$$


In the same manner, it is easy to prove that

$$
\|\vartheta-\bar{\vartheta}\| \leq \varepsilon_{1} \hat{C}_{\Psi}, \quad \hat{C}_{\Psi}=\frac{1}{(1-\nabla) \Gamma(q+1)}>0
$$

Therefore, from inequalities (20) and (21), we have

$$
\|(\theta, \vartheta)-(\bar{\theta}, \bar{\vartheta})\| \leq \max \left\{\varepsilon_{1} \hat{C}_{\Phi}, \varepsilon_{2} \hat{C}_{\Psi}\right\}=\varepsilon \hat{C}_{\Phi, \Psi}
$$

Therefore the solutions of a coupled system of BVPs (1) are Ulam-Hyers stable. Further, if $\Theta_{\Phi, \Psi}(\varepsilon)=\varepsilon$, hence (22) can be expressed as

$$
\|(\theta, \vartheta)-(\bar{\theta}, \bar{\vartheta})\| \leq \hat{C}_{\Phi, \Psi} \Theta_{\Phi, \Psi}(\varepsilon)
$$

Therefore, $\Theta_{\Phi, \Psi}(0)=0$ in (23) holds. Thus, solutions of the system of BVP (1) are generalized Ulam-Hyers stable. Thus we have investigated sufficient condition for Ulam-Hyers stability generalized Ulam-Hyers stability, that is, $\nabla<1$.

\section{Examples}

In this section, we provide some examples of the coupled system (1). We compute the iterative approximation for the extremal solution of the corresponding examples. We also provide error estimates for the extremal solutions. Furthermore, the stability analysis under the sufficient conditions for the examples of system (1) is also the concern of this section. We also provide the plots of each example of system (1) in the frame figures.

Example 5.1 Consider the following coupled system of BVPs of FDEs:

$$
\begin{aligned}
& \mathbb{D}^{\frac{5}{2}} \theta(\xi)+\frac{t^{\frac{7}{2}} \vartheta(\xi)\left(56,754 \vartheta^{2}(\xi)+160\right)}{1+\vartheta^{2}(\xi)}=0, \quad 0 \leq \xi \leq 1, \\
& \mathbb{D}^{\frac{27}{10}} \vartheta(\xi)+\frac{t^{\frac{9}{2}} \theta(\xi)(\theta(\xi)+1)\left(23,471 e^{(-\theta(\xi))}+120\right)}{2+\theta(\xi)}=0, \quad 0 \leq \xi \leq 1,
\end{aligned}
$$

under the boundary condition given by

$$
\theta(1)=\theta^{\prime}(0)=\theta^{\prime \prime}(0)=0, \quad \vartheta(1)=\vartheta^{\prime}(0)=\vartheta^{\prime \prime}(0)=0 .
$$

As for $\mathbb{A}=\mathbb{B}=0.0001$, we have $\nabla=7.2147 e^{-10}<1$. When $n=3$ is large enough, iterative sequences for the approximate minimal and maximal solutions $\theta_{n}$ and $\bar{\theta}_{n}^{*}$ respectively are:

$$
\begin{aligned}
& \theta^{*}(\xi)=\theta_{3}(\xi), \\
& \vartheta^{*}(\xi)=\int_{0}^{1} \mathbb{G}_{2}(\xi, \sigma) \frac{\sigma^{\frac{9}{2}} \theta_{2}(\sigma)\left(\theta_{2}(\sigma)+1\right)\left(23,471 e^{-\theta_{2}(\sigma)}+120\right)}{1+\theta_{2}(\sigma)} d \sigma \\
& \bar{\theta}^{*}(\xi)=\theta_{3}^{*}(\xi), \\
& \bar{\vartheta}^{*}(\xi)=\int_{0}^{1} \mathbb{G}_{2}(\xi, \sigma) \frac{\sigma^{\frac{9}{2}} \theta_{2}^{*}(\sigma)\left(\theta_{2}^{*}(\sigma)+1\right)\left(23,471 e^{-\theta_{2}^{*}(\sigma)}+120\right)}{1+\theta_{2}^{*}(\sigma)} d \sigma .
\end{aligned}
$$



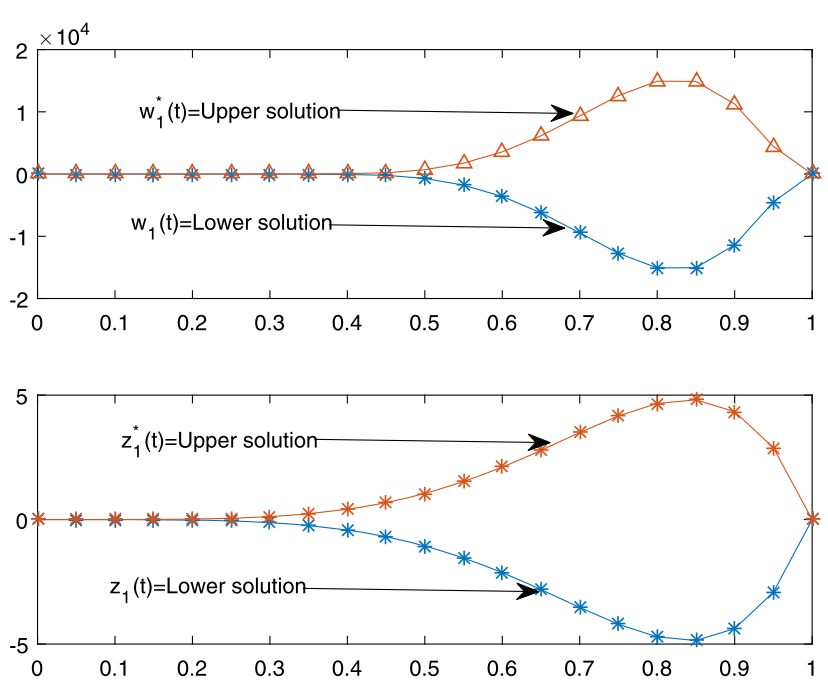

Figure 1 A line graph of the solutions of Example 5.1

Let $\left(\theta_{0}, \vartheta_{0}\right)=(-0.01,-0.01),\left(\theta_{0}^{*}, \vartheta_{0}^{*}\right)=(0.01,0.01)$ be the minimal and maximal solutions of Example 5.1 respectively, then we have their corresponding maximum error estimates:

$$
\begin{aligned}
e_{3} & =\left\|\theta-\theta_{3}\right\| \leq \frac{\nabla^{3}}{1-\nabla} \times e_{1} \leq 3.7554292227 e^{-28} \times \max _{\xi \in[0,1]}\left|\theta_{1}(\xi)+0.01\right| \\
& \simeq 5.66292592845 e^{-24}, \\
e_{3}^{*} & =\left\|\theta-\theta_{3}^{*}\right\| \leq \frac{\nabla^{3}}{1-\nabla} \times e_{1}^{*} \leq 3.7554292227 e^{-28} \times \max _{\xi \in[0,1]}\left|0.01-\theta_{1}^{*}(\xi)\right| \\
& \simeq 5.60233983913 e^{-24} .
\end{aligned}
$$

The iterative solutions of minimal and maximal of Example 5.1 are plotted in Figs. 1-2 wherein for the simple execution of Matlab code, we have replaced $(\theta, \vartheta)$ by $(w, z)$ and $\left(\theta^{*}, \vartheta^{*}\right)$ by $\left(w^{*}, z^{*}\right)$. Each plot in the figures has the demonstration of physical behavior of the approximate solutions.

Further, as $\nabla<1$, therefore, the Ulam-Hyers stability and generalized Ulam-Hyers stability are obvious. The stability of the iterative solutions can also be observed from Figs. 1-2.

Example 5.2 To strengthen our results, we provide another example as follows:

$$
\begin{aligned}
& \mathbb{D}^{\frac{19}{5}} \theta(\xi)+\sqrt{1+\vartheta^{2}(\xi)}\left(\vartheta^{1.1}(\xi)+\vartheta^{2.3}(\xi)\right)=0, \quad 0 \leq \xi \leq 1, \\
& \mathbb{D}^{\frac{7}{2}} \vartheta(\xi)+\sqrt{\theta(\xi)}\left(\theta^{2.3}(\xi)+\theta^{1.1}(\xi)\right)=0, \quad 0 \leq \xi \leq 1,
\end{aligned}
$$

subject to boundary conditions given as

$$
\theta(1)=\theta^{\prime}(0)=\theta^{\prime \prime}(0)=\theta^{\prime \prime \prime}(0)=0, \quad \vartheta(1)=\vartheta^{\prime}(0)=\vartheta^{\prime \prime}(0)=\vartheta^{\prime \prime \prime}(0)=0 .
$$



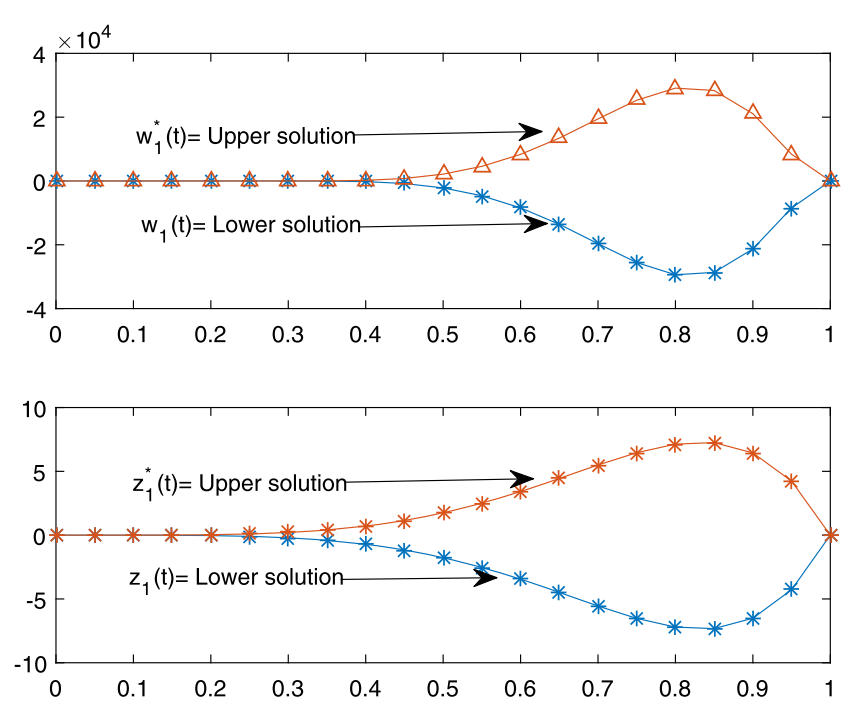

Figure 2 A line graph of the solutions of Example 5.1, when $p=\frac{11}{5}$ and $q=\frac{15}{7}$

As for $\mathbb{A}=\mathbb{B}=\frac{1}{64}$, we have $\nabla=1.1767 e^{-6}<1$. When $n=3$ is large enough, iterative sequences for the approximate minimal and maximal solutions $\theta_{n}$ and $\bar{\theta}_{n}^{*}$ respectively are:

$$
\begin{array}{ll}
\theta^{*}(\xi)=\theta_{3}(\xi), \quad \vartheta^{*}(\xi)=\int_{0}^{1} \mathbb{G}_{2}(\xi, \sigma) \sqrt{\theta_{2}(\sigma)}\left(\theta_{2}^{2.3}(\sigma)+\theta_{2}^{1.1}(\sigma)\right) d \sigma \\
\bar{\theta}^{*}(\xi)=\theta_{3}^{*}(\xi), \quad \bar{\vartheta}^{*}(\xi)=\int_{0}^{1} \mathbb{G}_{2}(\xi, \sigma) \sqrt{\theta_{2}^{*}(\sigma)}\left(\left(\theta_{2}^{*}(\sigma)\right)^{2.3}+\left(\theta_{2}^{*}(\sigma)\right)^{1.1}\right) d \sigma .
\end{array}
$$

Let $\left(\theta_{0}, \vartheta_{0}\right)=(-3,-3),\left(\theta_{0}^{*}, \vartheta_{0}^{*}\right)=(3,3)$ be the maximal and minimal solutions of Example 5.2 respectively, then the error estimates are:

$$
\begin{aligned}
e_{3} & =\left\|\theta-\theta_{3}\right\| \leq \frac{\nabla^{3}}{1-\nabla} \times e_{1} \leq 1.62914414119 e^{-18} \times \max _{\xi \in[0,1]}\left|\theta_{1}(\xi)+3\right| \\
& \simeq 5.03862710112 e^{-18}, \\
e_{3}^{*} & =\left\|\theta-\theta_{3}^{*}\right\| \leq \frac{\nabla^{3}}{1-\nabla} \times e_{1}^{*} \leq 1.62914414119 e^{-18} \times \max _{\xi \in[0,1]}\left|3-\theta_{1}^{*}(\xi)\right| \\
& \simeq 4.88743242358 e^{-18} .
\end{aligned}
$$

The iterative solutions of minimal and maximal of Example 5.2 are plotted in Figs. 3-4, wherein for the simple execution of Matlab code, we have replaced $(\theta, \vartheta)$ by $(w, z)$ and $\left(\theta^{*}, \vartheta^{*}\right)$ by $\left(w^{*}, z^{*}\right)$. Each plot in the figures has the demonstration of physical behavior of the approximate solutions.

Further, as $\nabla<1$, therefore, the Ulam-Hyers stability and generalized Ulam-Hyers stability are obvious. The stability of the iterative solutions can also be observed from Figs. 3-4. 

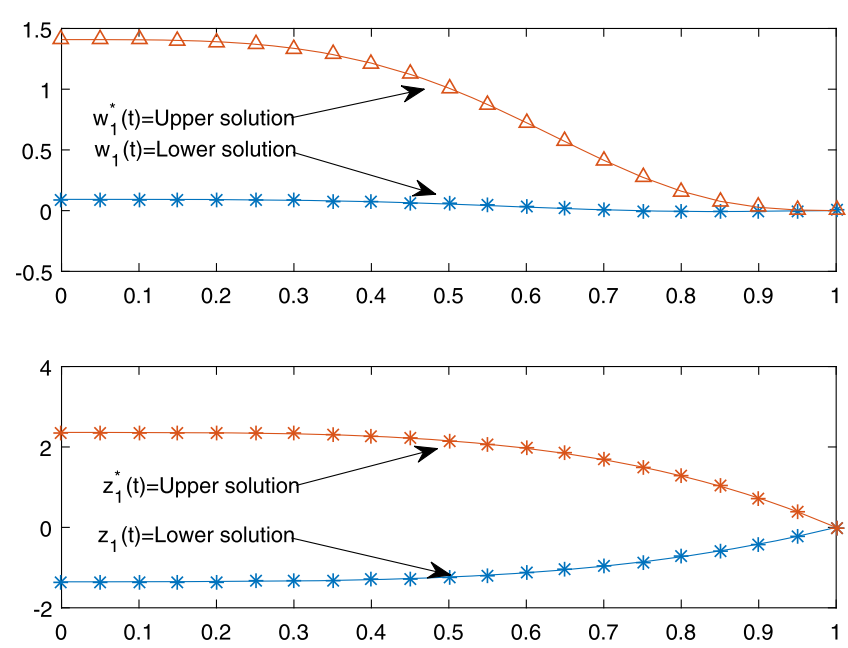

Figure $3 \mathrm{~A}$ line graph of the solutions of Example 5.2
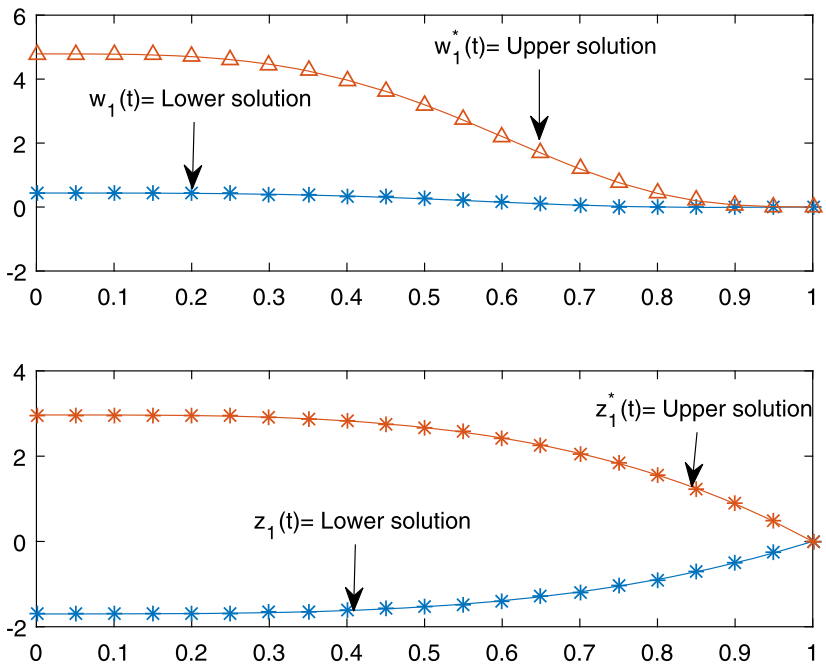

Figure 4 A line graph of the solutions of Example 5.2, when $p=\frac{17}{5}$ and $q=\frac{10}{3}$

Example 5.3 Consider the following coupled system of BVPs of FDEs:

$$
\begin{aligned}
& \mathbb{D}^{\frac{21}{5}} \theta(\xi)+\frac{t^{\frac{15}{4}} \vartheta(\xi)\left(\vartheta^{2}(\xi)+160\right)}{\left(1+\vartheta^{2}(\xi)\right)^{2}}=0, \quad 0 \leq \xi \leq 1, \\
& \mathbb{D}^{\frac{9}{2}} \vartheta(\xi)+\frac{t^{\frac{19}{4}} \theta(\xi)(\theta(\xi)+1)(\theta(\xi)+9)\left(e^{-2 \theta(\xi)}+120\right)}{(2+\theta(\xi))^{2}}=0, \quad 0 \leq \xi \leq 1,
\end{aligned}
$$

with the given boundary condition

$$
\begin{aligned}
& \theta(1)=\theta^{\prime}(0)=\theta^{\prime \prime}(0)=\theta^{\prime \prime \prime}(0)=\theta^{\prime \prime \prime \prime}(0)=0, \\
& \vartheta(1)=\vartheta^{\prime}(0)=\vartheta^{\prime \prime}(0)=\vartheta^{\prime \prime \prime}(0)=\vartheta^{\prime \prime \prime \prime}(0)=0 .
\end{aligned}
$$


Obviously, $\mathbb{A}=\mathbb{B}=\frac{1}{64}$, from which it is easy to see that $\nabla=1.4317 e^{-7}<1$. For taking $n=3$ is large enough, we have the iterative sequences for approximate minimal and maximal solutions $\theta_{n}$ and $\bar{\theta}_{n}^{*}$ as given by

$$
\begin{aligned}
& \theta^{*}(\xi)=\theta_{3}(\xi), \\
& \vartheta^{*}(\xi)=\int_{0}^{1} \mathbb{G}_{2}(\xi, \sigma) \frac{\sigma^{\frac{19}{4}} \theta_{2}(\sigma)\left(\theta_{2}(\sigma)+1\right)\left(\theta_{2}(\sigma)+9\right)\left(e^{-2 \theta_{2}(\sigma)}+120\right)}{\left(2+\theta_{2}(\sigma)\right)^{2}} d \sigma, \\
& \bar{\theta}^{*}(\xi)=\theta_{3}^{*}(\xi), \\
& \bar{\vartheta}^{*}(\xi)=\int_{0}^{1} \mathbb{G}_{2}(\xi, \sigma) \frac{t^{\frac{19}{4}} \theta_{2}^{*}(\sigma)\left(\theta_{2}^{*}(\sigma)+1\right)\left(\theta_{2}^{*}(\sigma)+9\right)\left(e^{-2 \theta_{2}^{*}(\sigma)}+120\right)}{\left(2+\theta_{2}^{*}(\sigma)\right)^{2}} d \sigma,
\end{aligned}
$$

respectively. Let $\left(\theta_{0}, \vartheta_{0}\right)=(-0.1,-0.1),\left(\theta_{0}^{*}, \vartheta_{0}^{*}\right)=(0.1,0.1)$ be maximal and minimal solutions respectively of Example 5.3, then

$$
\begin{aligned}
e_{3} & =\left\|\theta-\theta_{3}\right\| \leq \frac{\nabla^{3}}{1-\nabla} \times e_{1} \leq 2.93476154975 e^{-21} \times \max _{\xi \in[0,1]}\left|\theta_{1}(\xi)+0.1\right| \\
& \simeq 2.93476154975 e^{-22}, \\
e_{3}^{*} & =\left\|\theta-\theta_{3}^{*}\right\| \leq \frac{\nabla^{3}}{1-\nabla} \times e_{1}^{*} \leq 2.93476154975 e^{-21} \times \max _{\xi \in[0,1]}\left|0.1-\theta_{1}^{*}(\xi)\right| \\
& \simeq 2.93476154975 e^{-22} .
\end{aligned}
$$

Physical behavior of the approximate maximal and minimal solutions has also been demonstrated by providing Figs. 5-6, wherein, for the simple execution of Matlab code, we have replaced $(\theta, \vartheta)$ by $(w, z)$ and $\left(\theta^{*}, \vartheta^{*}\right)$ by $\left(w^{*}, z^{*}\right)$.

It is easy to obtain the conditions of various types of Ulam-Hyers stabilities for the solutions. The stability of iterative solutions is also obvious from Figs. 5-6.
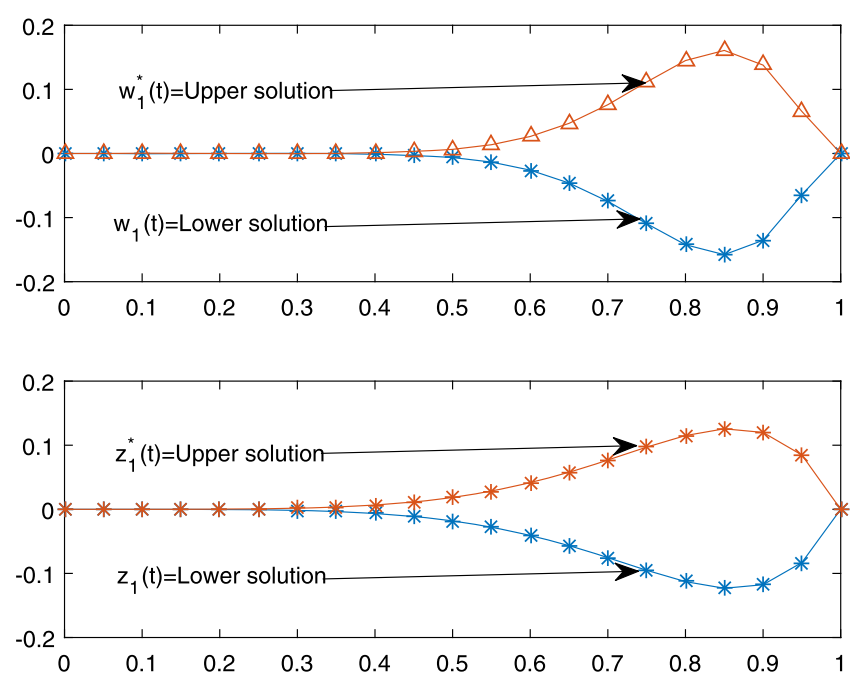

Figure $5 \mathrm{~A}$ line graph of the solutions of Example 5.3 

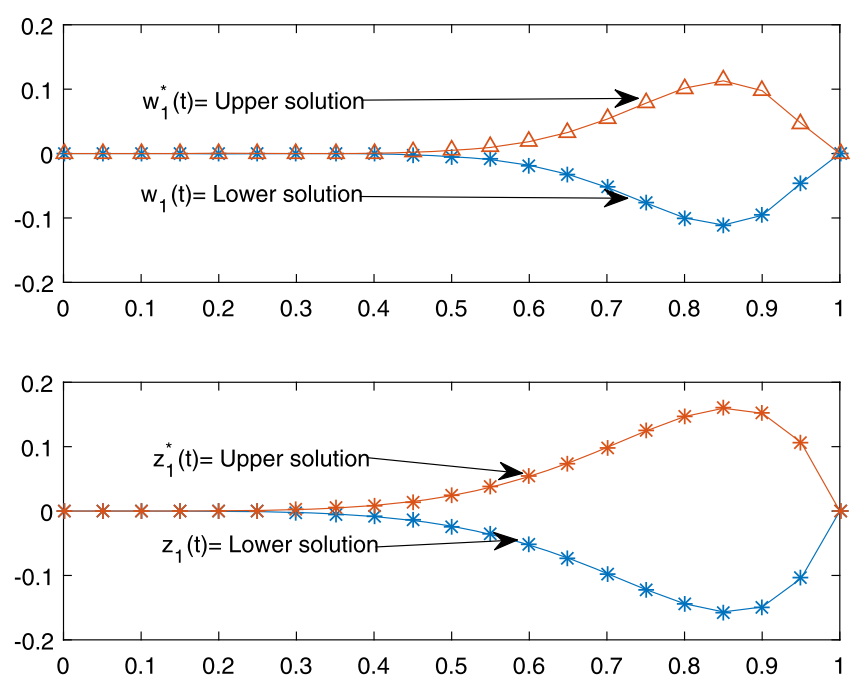

Figure 6 A line graph of the solutions of Example 5.3, when $p=\frac{23}{5}$ and $q=\frac{13}{3}$

Figures discussion: We have plotted the iterative solutions of three considered examples of system (1). These plots are provided in Figs. 1-6. For the sketch of plots, we have used the subplot command in the Matlab software. Each plot of numerical results shows a meaningful behavior of the extremal solutions of the coupled system of fractional differential equations. The different visualizations of each upper and lower solutions are very clear.

\section{Conclusion}

We have developed sufficient conditions for the existence of extremal solutions to a coupled system of FDEs with highier order boundary conditions. We have successfully provided these sufficient conditions on the basis of combining the method of upper and lower solutions together with the method of monotone iterative technique. We have constructed two monotonic sequences corresponding to upper and lower solutions such that a monotonically increasing sequence converged to the maximal solutions and the monotonically decreasing sequence converged to the minimal solutions of the system under consideration. Finally, an error estimate has been established, which provided the efficiency of the method.

Acknowledgements

Further we are thankful to the anonymous referee for useful suggestions.

\section{Funding}

The second author would like to thank Prince Sultan University for funding this work through research group Nonlinear Analysis Methods in Applied Mathematics (NAMAM), group number RG-DES-2017-01-17.

\section{Abbreviations}

FDEs; BVPS

Availability of data and materials

Not applicable. 
Authors' contributions

All authors have done equal contribution in this article. All authors read and approved the final manuscript.

\section{Author details}

'Department of Mathematics, Abdul Wali Khan University Mardan, Khyber Pakhtunkhwa, Pakistan. ${ }^{2}$ Department of Mathematics and General Sciences, Prince Sultan University, Riyadh, Saudi Arabia. ${ }^{3}$ Department of Mathematics, University of Malakand, Khyber Pakhtunkhwa, Pakistan. ${ }^{4}$ Department of Mathematics, Çankaya University, Ankara, Turkey.

\section{Publisher's Note}

Springer Nature remains neutral with regard to jurisdictional claims in published maps and institutional affiliations.

\section{Received: 6 February 2019 Accepted: 21 May 2019 Published online: 30 May 2019}

\section{References}

1. Kilbas, A.A., Marichev, O.I., Samko, S.G.: Fractional Integrals and Derivatives (Theory and Applications). Gordon and Breach, Switzerland (1993)

2. Miller, K.S., Ross, B.: An Introduction to the Fractional Calculus and Fractional Differential Equations. Wiley, New York (1993)

3. Podlubny, I.: Fractional Differential Equations, Mathematics in Science and Engineering. Academic Press, New York (1999)

4. Hilfer, R.: Applications of Fractional Calculus in Physics. World Scientific, Singapore (2000)

5. Kilbas, A.A., Srivastava, H.M., Trujillo, J.J.: Theory and Applications of Fractional Differential Equations. Elsevier, Amsterdam (2006)

6. Benchohra, M., Graef, J.R., Hamani, S.: Existence results for boundary value problems with nonlinear fractional differential equations. Appl. Anal. 87, 851-863 (2008)

7. Agarwal, R.P., Belmekki, M., Benchohra, M.: A survey on semilinear differential equations and inclusions involving Riemann-Liouville fractional derivative. Adv. Differ. Equ. 2009, Article ID 981728 (2009)

8. Agarwal, R.P., Benchohra, M., Hamani, S.: A survey on existence results for boundary value problems of nonlinear fractional differential equations and inclusions. Acta Appl. Math. 109, 973-1033 (2012)

9. Abbas, S., Benchohra, M.: Topics in Fractional Differential Equations. Springer, Berlin (2012) 12 pages

10. Benchohra, M., Graef, J.R., Hamani, S.: Existence results for boundary value problems with non-linear fractional differential equations. Appl. Anal. 87(7), 851-863 (2008)

11. Isaia, F.: On a nonlinear integral equation without compactness. Acta Math. Univ. Comen. iv, 233-240 (2006)

12. Kilbas, A.A., Marichev, O.I., Samko, S.G.: Fractional Integrals and Derivatives (Theory and Applications). Gordon and Breach, Switzerland (1993)

13. Miller, K.S., Ross, B.: An Introduction to the Fractional Calculus and Fractional Differential Equations. Wiley, New York (1993)

14. Timoshenko, S.P.: Theory of Elastic Stability. McGraw-Hill, New York (1961)

15. Soedel, W.: Vibrations of Shells and Plates. Dekker, New York (1993)

16. Yang, W.: Positive solution to nonzero boundary values problem for a coupled system of nonlinear fractional differential equations. Comput. Math. Appl. 63, 288-297 (2012)

17. Wang, J., Xiang, H., Liu, Z.: Positive solution to nonzero boundary values problem for a coupled system of nonlinear fractional differential equations. Int. J. Differ. Equ. 2010, Article ID 186928 (2010)

18. Su, X.: Boundary value problem for a coupled system of nonlinear fractional differential equations. Appl. Math. Lett. 22, 64-69 (2009)

19. Wang, G., Agarwal, R.P., Cabada, A.: Existence results and the monotone iterative technique for systems of nonlinear fractional differential equations. Appl. Math. Lett. 25(6), 1019-1024 (2012)

20. Zeidler, E.: Nonlinear Functional Analysis and Its Applications, Part II/B: Nonlinear Monotone Operators. Springer, New York (1989)

21. Shah, K., Khan, R.A.: Iterative solutions to a coupled system of nonlinear fractional differential equations. J. Fract. Calc. Appl. 7(2), 40-50 (2016)

22. Shah, K., Khalil, H., Khan, R.A.: Upper and lower solutions to a coupled system of nonlinear fractional differential equations. Prog. Fract. Differ. Appl. 1(1), 1-10 (2016)

23. Ahmad, B., Nieto, J.J.: Existence results for a coupled system of nonlinear fractional differential equations with three-point boundary conditions. Comput. Math. Appl. 58, 1838-1843 (2009)

24. Gafiychuk, V., Datsko, B., Meleshko, V., Blackmore, D.: Analysis of the solutions of coupled nonlinear fractional reaction-diffusion equations. Chaos Solitons Fractals 41, 1095-1104 (2009)

25. Arshad, A., Shah, K., Jarad, F., Gupta, V., Abdeljawad, T.: Existence and stability analysis to a coupled system of implicit type impulsive boundary value problems of fractional-order differential equations. Adv. Differ. Equ. 2019, 101 (2019)

26. Jarad, F., Abdeljawad, T., Hammouch, Z.: On a class of ordinary differential equations in the frame of Atangana-Baleanu fractional derivative. Chaos Solitons Fractals 117, 16-20 (2019)

27. Gambo, Y.Y., Ameen, R., Jarad, F., Abdeljawad, T.: Existence and uniqueness of solutions to fractional differential equations in the frame of generalized Caputo fractional derivatives. Adv. Differ. Equ. 2018, 134 (2018)

28. Ameen, R., Jarad, F., Abdeljawad, T.: Ulam stability for delay fractional differential equations with a generalized Caputo derivative. Filomat 32(15), 5265-5274 (2018)

29. Adjabi, Y., Jarad, F., Baleanu, D., Abdeljawad, T.: On Cauchy problems with Caputo Hadamard fractional derivatives. J. Comput. Anal. Appl. 21(4), 661-681 (2016)

30. Abdeljawad, T., Jarad, F., Baleanu, D.: On the existence and the uniqueness theorem for fractional differential equations with bounded delay within Caputo derivatives. Sci. China Ser. A, Math. 51(10), 1775-1786 (2008)

31. Abdeljawad, T., Baleanu, D., Jarad, F.: Existence and uniqueness theorem for a class of delay differential equations with left and right Caputo fractional derivatives. J. Math. Phys. 49(8), Article ID 083507 (2008) 
32. Ladde, G.S., Lakshmikantham, V., Vatsala, A.S.: Monotone Iterative Technique for Nonlinear Differential Equations. Pitman Publishing Inc., Boston (1985)

33. Bai, C., Fang, J.: The existence of a positive solution for a singular coupled system of nonlinear fractional differential equations. Comput. Math. Appl. 150,611-621 (2004)

34. Zhang, S: Monotone iterative method for initial value problem involving Riemann-Liouville fractional derivatives Nonlinear Anal., Theory Methods Appl. 71(5), 2087-2093 (2009)

35. Mcrae, F.A.: Monotone iterative technique and existence results for fractional differential equations. Nonlinear Anal., Theory Methods Appl. 71(12), 6093-6096 (2009)

36. Al-Refai, M., Ali Hajji, M.: Monotone iterative sequences for nonlinear boundary value problems of fractional order. Nonlinear Anal., Theory Methods Appl. 74(11), 3531-3539 (2011)

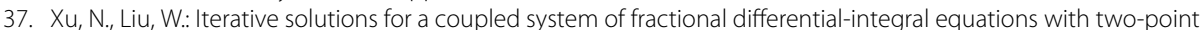
boundary conditions. Appl. Math. Comput. 244, 903-911 (2014)

38. Liu, X., Jia, M.: Multiple solutions for fractional differential equations with nonlinear boundary conditions. Comput. Math. Appl. 59, 2880-2886 (2010)

39. He, Z., He, X.: Monotone iterative technique for impulsive integro-differential equations with periodic boundary conditions. Comput. Math. Appl. 48, 73-84 (2004)

40. De Coster, C., Habets, P.: Two-Point Boundary Value Problems: Lower and Upper Solutions. Elsevier, Amsterdam (2006)

41. Li, F., Jia, M., Liu, X., Li, C., Li, G.: Existence and uniqueness of solutions of second-order three-point boundary value problems with upper and lower solutions in the reverse order. Nonlinear Anal., Theory Methods Appl. 68, 2381-2388 (2008)

42. Hirstova, S., Tunç, C.: Stability of nonlinear Volterra integro-differential equations with Caputo fractional derivative and bounded delays. Electron. J. Differ. Equ. 30, 1 (2019)

43. Ali, S., Shah, K., Jarad, F.: On stable iterative solutions for a class of boundary value problem of nonlinear fractional order differential equations. Math. Methods Appl. Sci. 42(3), 969-981 (2019)

44. Rus, I.A.: Ulam stabilities of ordinary differential equations in a Banach space. Carpath. J. Math. 26, 103-107 (2010)

45. Rassias, T.M.: On the stability of the linear mapping in Banach spaces. Proc. Am. Math. Soc. 72, 297-300 (1978)

46. Rassias, T.M.: On the stability of functional equations and a problem of Ulam. Acta Appl. Math. 26, 23-130 (2000)

47. Hirstova, S., Tunç, C.: Stability of nonlinear Volterra integro-differential equations with Caputo fractional derivative and bounded delays. Electron. J. Differ. Equ. 30, 1 (2019)

48. Wang, J., Fečkan, M., Zhou, Y.: Fractional order differential switched systems with coupled nonlocal initial and impulsive conditions. Bull. Sci. Math. 141, 727-746 (2017)

49. Cabada, A., Wang, G.: Positive solutions of nonlinear fractional differential equations with integral boundary value conditions. J. Math. Anal. Appl. 389, 403-411 (2012)

\section{Submit your manuscript to a SpringerOpen ${ }^{\circ}$ journal and benefit from:}

- Convenient online submission

- Rigorous peer review

- Open access: articles freely available online

- High visibility within the field

- Retaining the copyright to your article

Submit your next manuscript at $>$ springeropen.com 